\title{
Anthelmintic resistance and the control of worms
}

\begin{abstract}
Many people, particularly in the tropics, harbour parasitic worms, usually to the detriment of their health, and sometimes with devastating effect. Helminths also have an enormous economic impact in animal husbandry. Therefore, it is surprising and worrying that resistance to the relatively small number of anthelmintic drugs that are available has received much less attention than resistance to antibacterial or other antiinfective agents, particularly with respect to human helminths $[1,2]$.
\end{abstract}

The major issue in the development of resistance is the percentage contribution those organisms surviving therapy make to the next generation. With few exceptions, parasitic helminths do not multiply in their mammalian hosts. Therefore, resistance is unlikely to become a problem unless there is systematic mass therapy, and provided a reservoir of infective stages that have not encountered the drug remains as the source of new infections.

The impact of mass treatment is illustrated by reference to grazing animals in parts of the UK in which nematodes are a serious problem in young lambs, causing reduced growth or even death. To prevent pasture contamination farmers treat the whole flock, including the ewes. Unless animals continue to graze on pasture already heavily contaminated with susceptible worms, the next generation of infections will come from those surviving therapy. Failure to grasp this has led to widespread benzimidazole resistance and spreading levamisole resistance [3]. In contrast it has been traditional only to treat first year dairy calves. Second year heifers and adults continue to shed some nematode eggs and are the main source of infection on the farm. Up to 1997 no resistant nematodes had been reported in cattle in the UK, although this situation can be expected to change with greater use of anthelmintics [4].

Cyathostomes are a serious cause of disease in horses. Since many horses are valuable animals, they are treated regularly with anthelmintics. The result is widespread resistance to benzimidazoles and, at least in the USA, resistance to pyrantel $[5,6]$. Work in Australia suggests that drought encourages the development of resistance by killing off free-living stages in the environment so that the next generation of infections come from worms surviving treatment [7]. This is an important warning for those treating human communities for control of nematodes in the tropics during the dry season.

Antiparasitic drugs make up nearly $30 \%$ of the animal health business in the UK. The success of chemotherapy may explain why research in veterinary parasitology has been poorly funded in recent years, with major cutbacks in Australia, the UK and the USA [8]. Ironically, this has occurred as drug resistance is becoming a serious threat to production, particularly to the sheep industry. Although by human helminthological standards there is a large bibliography on drug resistance in veterinary helminths [e.g., 9, 10], the paucity of funding has resulted in a lack of sensitive tests to detect resistance, limited information on the epidemiology of resistance and few proven strategies for prolonging the effective life of drugs [11].

There are no in-vitro tests for detecting resistance in trematodes or cestodes, although tests for praziquantel resistance in Schistosoma mansoni are being developed. Three types of test are used to detect resistance in veterinary nematodes: faecal egg count reduction, egg hatch and larval development. The faecal egg count reduction tests determine the numbers of eggs in a group of animals at the time of dosing and 10-14 days later (i.e., less than the prepatent period) and could easily be applied to groups of infected human beings. However, ivermectin can sterilise female nematodes for at least 14 days [12]. If re-infection occurs before nematodes surviving therapy resume egg laying it is impossible to distinguish between resistance and reinfection, unless re-infection is prevented. Further research is needed into this problem. The egg hatch test relies on the ability of eggs from benzimidazoleresistant nematodes to develop and hatch in the presence of appropriate concentrations of a representative benzimidazole, thiabendazole, while susceptible eggs die [13]. The test may be applicable to human hookworms but has not been adequately validated. The larval development test, in which larvae develop in the presence of anthelmintic and a food source [14], has yet to be applied to those human nematodes that have a free living feeding stage.

Low-level resistance to benzimidazoles in certain veterinary nematodes is associated with a change in amino acid 200 of $\beta$-tubulin from phenylalanine to tyrosine [15]. Whether this always occurs in benzimidazole resistance is not known, but PCR-based tests to 
examine the frequency of occurrence of this mutation in human helminths would be useful. Levamisole resistance is associated with reduced drug binding to the acetylcholine receptor [16] through an undefined molecular change or changes. The mechanism of resistance to ivermectin is still unknown, and more than one mechanism of resistance may have developed. The potential for the development of resistance to ivermectin in nematodes is very important as it is crucial to onchocerciasis control in Africa. The lack of activity against adult worms could indicate that it would be relatively easy for larval stages to alter expression of the target site and thus develop resistance.

There are many chemotherapeutic trials in humans reported in the literature that show differing efficacies of the same drug. This is a cause for concern because it could indicate that natural tolerance to anthelmintic agents exists in some populations of nematodes and, with mass therapy, full resistance could develop rapidly. Two well-conducted trials on hookworms have given good evidence for benzimidazole resistance in Necator americanus in West Africa [17] and pyrantel resistance in Ancylostoma duodenale in Australia [18]. Without new anthelmintics hookworms could become difficult to treat.

Systematic mass therapy of infection with human schistosomes is not widely used and resistance has not become a practical issue, although individual cases of resistance to oxamniquine have been known for several years. Because of its excellent activity praziquantel is now the preferred drug and has been used heavily in China and Egypt. The first signs of resistance to praziquantel are emerging in Egypt, where a small percentage of patients do not respond to therapy and isolates tested in mice also show resistance [19]. There is no simple test for resistance to praziquantel yet available and the molecular basis of resistance has not been established, consequently the full extent of resistance remains unknown. Little research is being undertaken to find a successor to praziquantel and the current reliance on one drug is of concern.

Infection with the liver fluke, Fasciola hepatica, is a zoonosis and, where transmission from animals to humans occurs via the snail host, the outlook for chemical control is not encouraging. Rabbits act as an untreated reservoir of infection in the UK and it might be thought that as a result resistance would be slow to develop. The most useful antitrematodal drug, praziquantel, is not effective against $F$. hepatica and a veterinary compound, triclabendazole, is the most active compound available. Resistance to triclabendazole has been reported in sheep in Australia [20], Ireland and Scotland. Resistance to some other fasciolicides has also been found in animals, but owing to lack of tests, there is no information on the extent of the problem anywhere in the world. Unfortunately resistance can only be confirmed by passage through snails, infection of rodents or sheep, and subsequent treatment of the infected animals. How quickly resistance will develop and spread is unknown, but it cannot be long before treatment failures caused by drug resistance are found in human patients.

The only serious diseases caused by tapeworms in man are from larval stages of Taenia solium, Echinococcus granulosus and E. multilocularis. There has been one report of development of resistance to albendazole in an experimental infection with larval E. granulosus [21] and it seems possible that such a situation could develop in clinical cases. Adult tapeworms might develop resistance to praziquantel, but there have been no reports of research into this possibility.

All who treat patients infected with parasitic helminths recognise that low efficacy of anthelmintics can result from inappropriate choice of drug, failure of patient compliance and pharmacokinetic differences between patients. Low efficacy may also be due to drug resistance. However, proving that failure is due to resistance remains problematic.

GERALD C. COLES Department of Clinical Veterinary Science, University of Bristol, Langford House, Bristol BS40 5DU. E-mail: gerald.c.coles@bristol.ac.uk

\section{References}

1. Coles GC. Chemotherapy of human nematodes: learning from the problems in sheep. $J R$ Soc Med 1995; 88: 649-651P.

2. Geerts S, Coles GC, Gryseels B. Anthelmintic resistance in human helminths: learning from the problems with worm control in livestock. Parasitol Today 1997; 13: 149-151.

3. Hong C, Hunt KR, Coles GC. Occurrence of anthelmintic resistant nematodes on sheep farms in England and goat farms in England and Wales. Vet Rec 1996; 139: 83-86.

4. Coles GC, Stafford KA, MacKay PH. Ivermectin-resistant Cooperia species from calves on a farm in Somerset. Vet Rec 1998; 142: 255-256.

5. Bauer C. Anthelmintic resistance in nematodes of horses. In: Coles GC, Borgsteede FHM, Geerts S (eds) Anthelmintic resistance in nematodes of farm animals. Brussels, European Commission. 1994: 17-24.

6. Chapman MR, French DD, Monahan CM, Klei TR. Identification and characterization of a pyrantel pamoate resistant cyathostome population. Vet Parasitol 1996; 66: 205-212.

7. Besier RB. Ecological selection for anthelmintic resistance: reevaluation of sheep worm control programs. In: van Wyk JA, van Schalkwyk PC (eds) Managing anthelmintic resistance in endoparasites. World Association for the Advancement of Veterinary Parasitology. 1997: 30-38.

8. Coles C, Klei TR. Animal parasites, politics and agricultural research. Parasitol Today 1995; 11: 276-278.

9. Taylor MA. Anthelmintic resistance in helminth parasites of domestic animals. Agr Zool Rev 1991; 5: 1-50.

10. Conder GA, Campbell WC. Chemotherapy of nematode infections of veterinary importance, with special reference to drug resistance. Adv Parasitol. 1995; 35: 1-84.

11. Coles GC, Roush RT. Slowing the spread of anthelmintic resistant nematodes of sheep and goats in the United Kingdom. 
Vet Rec 1992; 130: 505-510.

12. Jackson F. Anthelmintic resistance - the state of play. Br Vet $J$ 1993; 149: 123-138.

13. Coles GC, Bauer C, Borgsteede FHM et al. World Association for the Advancement of Veterinary Parasitology (WAAVP) methods for the detection of anthelmintic resistance in nematodes of veterinary importance. Vet Parasitol 1992; 44: $35-44$.

14. Coles GC. Tritschler JP, Giordano DJ, Laste NJ, Schmidt AL. Larval development test for detection of anthelmintic resistant nematodes. Res Vet Sci 1988; 45: 50-53.

15. Kwa MS, Veenstra JG, Roos MH. Benzimidazole resistance in Haemonchus contortus is correlated with a conserved mutation at amino acid 200 in $\beta$-tubulin isotype 1. Mol Biochem Parasitol 1994; 63: 299-303.

16. Moreno-Guzman MJ, Coles GC, Jimenez-Gonzalez A et al. Levamisole binding sites in Haemonchus contortus. Int $J$ Parasitol 1998; 28: 413-418.
17. De Clercq D, Sacko M, Behnke J, Gilbert F, Dorny P, Vercruysse J. Failure of mebendazole in treatment of human hookworm infections in the southern region of Mali. Am J Trop Med Hyg 1997; 57: 25-30.

18. Reynoldson JA, Behnke JM, Pallant LJ et al. Failure of pyrantel in treatment of human hookworm infections (Ancylostoma duodenale) in the Kimberley region of north west Australia. Acta Trop 1997; 68: 301-312.

19. Ismail M, Metwally A, Farghaly A, Bruce J, Tao L-F, Bennett JL. Characterization of isolates of Schistosoma mansoni from Egyptian villagers that tolerate high doses of praziquantel. $\mathrm{Am}$ $J$ Trop Med Hyg 1996; 55: 214-218.

20. Boray JC. Chemotherapy of infections with fasciolidae. In: Boray JC (ed) Immunology, pathobiology and control of Fasciolosis. Rahway, MSD AGVET, 83-97.

21. Morris DL, Taylor DH. Echinococcus granulosus: development of resistance to albendazole in an animal model. $J$ Helminthol 1990; 64: 171-174. 